\title{
Catalytic Intramolecular Aminoarylation of Unactivated Alkenes with Aryl Sulfonamides
}

Efrey A. Noten, Rory C. McAtee, and Corey R. J. Stephenson*

University of Michigan, Department of Chemistry, Willard Henry Dow Laboratory, 930 North University Ave., Ann Arbor MI 48109 United States.

Abstract: Arylethylamines are abundant motifs in myriad natural products and pharmaceuticals, so efficient methods to synthesize them are valuable in drug discovery. In this work, we disclose an intramolecular alkene aminoarylation cascade that exploits the electrophilicity of a nitrogencentered radical to form a $\mathrm{C}-\mathrm{N}$ bond, then repurposes the nitrogen atom's sulfonyl activating group as a traceless linker to form a subsequent $\mathrm{C}-\mathrm{C}$ bond. This photoredox catalysis protocol enables the preparation of densely substituted arylethylamines from commercially abundant aryl sulfonamides under mild conditions. Reaction optimization, scope, mechanism, and synthetic applications are discussed.

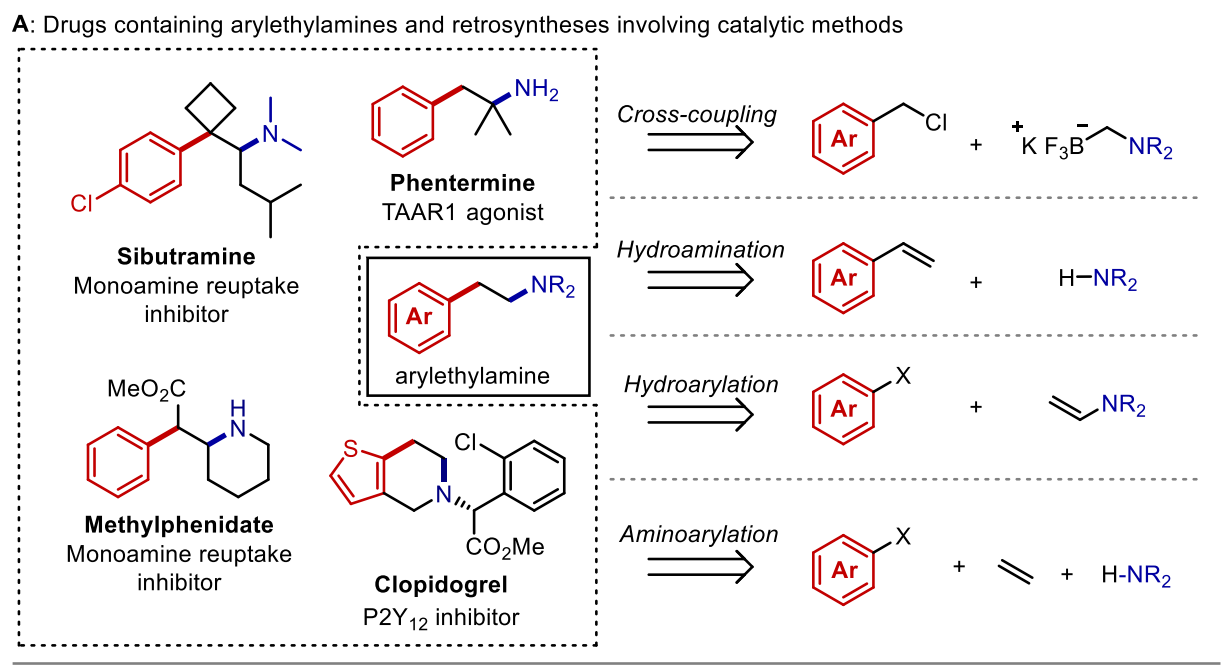

B: Our previous work: aminoarylation of activated alkenes through radical cations

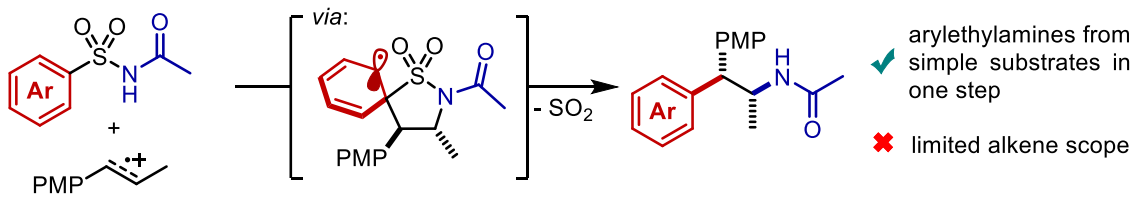

C: This work: aminoarylation of unactivated alkenes through $N$-centered radicals

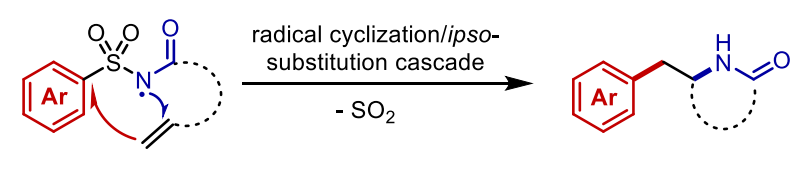

linear, achiral substrates $\cdot$ complex lactam products $\cdot 30$ examples

Design challenges:

- Dearomatization

- Hydroamination

- Phenol formation

Figure 1. A: Selected biologically active molecules containing arylethylamines and recent catalytic disconnections. B: Summary of our group's prior work on aminoarylation through alkene radical cations. C: Abstract depiction of aminoarylation cascade in the present work and challenges that were overcome 


\section{Introduction.}

The arylethylamine pharmacophore is conserved across a range of biologically active natural products and drugs, particularly in molecules that act on the central nervous system (Figure 1A, left). ${ }^{1}$ Conventional preparations of arylethylamines rely on linear, stoichiometric transformations to forge key $\mathrm{C}-\mathrm{C}$ and $\mathrm{C}-\mathrm{N}$ bonds. Such routes lack the combinatorial flexibility favored in early-stage medicinal chemistry campaigns and they restrict the accessible substitution patterns of the ethylene linker fragment. Substituents on the linker can drastically alter the molecule's lipophilicity, conformation, and elimination half-life. ${ }^{2,3}$ Modular preparations of complex arylethylamines from commercially available or easily synthesized substrates are therefore highly valuable, and considerable efforts have focused on this need (Figure 1A, right).

Recently, Murphy, Barrett, and coworkers published a method for arylethylamine synthesis by palladium-catalyzed $\mathrm{Csp}^{3}-\mathrm{Csp}^{3}$ cross-coupling of (chloromethyl)aryl electrophiles and aminomethyltrifluoroborate salts. ${ }^{4}$ A diverse library of compounds could be quickly produced in this manner; however, no products bearing linker substituents were reported. An alternative and succinct disconnection of an arylethylamine could be the difunctionalization of an alkene to incorporate (1) the $\mathrm{C}-\mathrm{N}$ bond, (2) the aryl- $\mathrm{Csp}^{3}$ bond, or (3) both bonds at once. The first case describes anti-Markovnikov hydroamination of a styrene, and many methods exist to accomplish this transformation effectively with the aid of photoredox, lanthanide, or transition metal catalysts. $^{5-8}$ The second case necessitates anti-Markovnikov hydroarylation of an enamine, which was only recently reported in good yields by Jui and coworkers. ${ }^{9}$ The third case entails aminoarylation of an unactivated alkene and is, in principle, the most modular of the three difunctionalization strategies. Because the substrate is decoupled from both the arene and the nitrogen atom, simple alkenes can be converted to arylethylamines in one step. Our interests in complex molecule synthesis by radical methods led us to question whether aminoarylation could be achieved with nitrogen-centered radicals. We perceived the advances by Knowles and coworkers in catalytic $\mathrm{N}$-centered radical generation as particularly enabling towards this goal. ${ }^{10}$ ${ }^{11}$ Formal homolysis of $\mathrm{N}-\mathrm{H}$ bonds via multiple-site concerted proton-electron transfer (MS-CPET) permits useful reactivity of $\mathrm{N}$-centered radicals without the need for harsh oxidants or strong 
bases. $^{12}$ If the $\mathrm{N}-\mathrm{H}$ bond is sufficiently acidic, stepwise deprotonation/oxidation sequences can also give $\mathrm{N}$-centered radicals under mild conditions. ${ }^{13}$

We first considered the state of the art in unactivated alkene aminoarylation to inform our reaction design. Varied tactics exist to construct the $\mathrm{C}-\mathrm{N}$ bond, but the $\mathrm{C}-\mathrm{C}$ bond is typically formed via reductive elimination of the aryl and alkyl fragments from a high-valent transition metal complex. Palladium-catalyzed alkene aminoarylation was explored extensively by Wolfe and coworkers in the preparation of saturated nitrogen heterocycles. ${ }^{14}$, 15 Engle and coworkers employed directing groups to orchestrate palladium- and nickel-catalyzed intermolecular aminoarylations of $\beta, \gamma$-unsaturated enamides and of homoallylic alcohols, respectively. ${ }^{16,17}$ Molander and coworkers merged photoredox- and transition metal catalysis by trapping amidyl radical cyclization intermediates with nickel to accomplish $\mathrm{C}-\mathrm{C}$ cross-coupling. ${ }^{18}$ We envisioned a desulfonylative 1,4-aryl migration (Smiles-Truce rearrangement) as an unconventional disconnection of the $\mathrm{C}-\mathrm{C}$ bond that could be induced by an $\mathrm{N}$-centered sulfonamidyl radical addition to an alkene. This aryl migration strategy would allow expedient entry to the arylethylamine scaffold from inexpensive sulfonamides. ${ }^{19}{ }^{20}$ Mechanistically, this distinct cascade would not require a cross-coupling catalyst and would grant access to sterically congested products that are challenging to prepare through transition metal-mediated methods. Although Molander's approach to aminoarylation initiates by an N-centered radical cyclization, the MSCPET method chosen to generate the radical necessitates $N$-aryl amide precursors. Oxidative cleavage of the auxiliary arene in the product is therefore necessary to provide the free lactam. By contrast, the designed desulfonylative aryl migration in this work would function as an in situ deprotection of the nitrogen atom.

We previously reported an alkene aminoarylation that proceeded through alkene radical cation intermediates. ${ }^{21}$ These electrophilic species successfully coupled with sulfonamides, leading to a Smiles-Truce rearrangement that delivered the desired arylethylamine (Figure 1B). However, only electron-rich, 1,2-disubstituted styrenes gave good yields. This restriction was attributed to the low oxidation potentials of the activated alkenes (1.28 V vs. SCE in $\mathrm{CH}_{3} \mathrm{CN}$ for trans-anethole) and the resistance of the corresponding radical cations to oligomerization. ${ }^{22,}{ }^{23}$ We expected our $\mathrm{N}$-centered radical approach to circumvent this limitation as well, based on strong literature 
precedent describing anti-Markovnikov sulfonamidyl radical additions to unactivated alkenes. ${ }^{24-}$ 30 However, no examples of Smiles-Truce rearrangements have been demonstrated in these systems.

We hypothesized that a second electron-withdrawing group on the nitrogen atom could convert the sulfonamide into a better leaving group. This modification would also prevent a reactive free amine from forming after $N$-desulfonylation, and it would further increase the acidity of the $\mathrm{N}-\mathrm{H}$ bond $\left(\mathrm{pKa} \approx 5\right.$ ) such that stepwise $\mathrm{N}$-centered radical generation could be feasible. ${ }^{31}$ However, intermolecular addition of $N$-acylsulfonamidyl radicals to unactivated alkenes was not observed. 1,4-aryl migration to the carbonyl oxygen instead gave desulfonylated phenols. To avert this undesired rearrangement, we synthesized $N$-acylsulfonamides bearing tethered alkenes that would rapidly trap the $\mathrm{N}$-centered radical in a 5-exo-trig cyclization. Desulfonylative aryl migration to the incipient alkyl radical would then provide the desired arylethylamine (Figure 2, right).

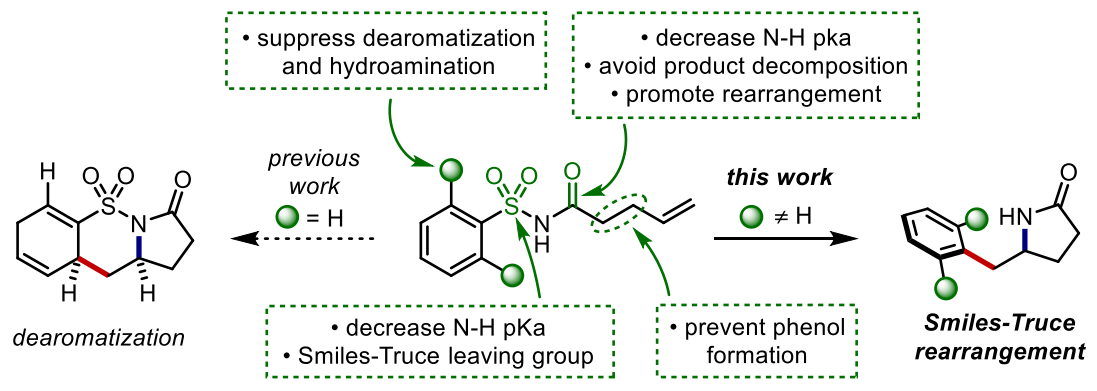

Figure 2. Structural features of the aminoarylation substrates that favor Smiles-Truce rearrangement and disfavor undesired side reactions.

Our initial efforts to develop this reaction revealed that instances of the substrates with only meta or para substitution would selectively undergo dearomative addition of the alkyl radical ortho to the sulfonyl group, followed by radical-polar crossover and protonation to garner 1,4cyclohexadiene-fused sultams (Figure 2, left). ${ }^{32}$ We reasoned that substituents occupying the ortho positions could inhibit this dearomative cyclization. Thus, when 2,6-difluorobenzenesulfonyl enamide 1d was exposed to the optimized dearomatization conditions from our previous work, the Smiles-Truce rearrangement occurred instead to give lactam 2d in 45\% isolated yield. Compound $\mathbf{1 d}$ was therefore chosen as a model substrate for the ensuing reaction optimization, and key observations from this process are highlighted in Table 1. 


\begin{tabular}{|cccccc} 
solvent (concentration), temperature \\
blue LEDs, 16-18 hr
\end{tabular}

Table 1. Selected optimization trials (see Supplementary Information for complete details). Reactions were conducted on a $0.1 \mathrm{mmol}$ scale in vials with $10 \mathrm{~mm}$ external diameter unless otherwise noted. Yields were determined by ${ }^{19} \mathrm{~F}$ NMR integration relative to 1.0 equiv. 4-fluorobromobenzene as an internal standard. Asterisks $\left({ }^{\star}\right)$ denote isolated yields. ${ }^{[a]}$ Reaction conducted in a $17 \mathrm{~mm}$ diameter vial.

\section{Reaction Optimization}

Although ${ }^{\mathrm{t}} \mathrm{BuOH}$ was beneficial for the dearomative cyclization as part of a binary solvent mixture with $\mathrm{PhCF}_{3}$, yield of $\mathbf{2} \mathbf{d}$ improved when ${ }^{t} \mathrm{BuOH}$ was excluded (entry 2). The strong oxidizing properties of Ir-1 were crucial; less oxidizing iridium photocatalysts such Ir-2 and Ir-3 gave reduced yields. At ambient temperature, $\mathrm{CH}_{3} \mathrm{CN}$ was found to give the highest yield of $\mathbf{2} \mathbf{d}$ among 
all solvents evaluated (entry 4). However, at $60{ }^{\circ} \mathrm{C}$, the yield decreased in $\mathrm{CH}_{3} \mathrm{CN}$ but improved further in $\mathrm{PhCF}_{3}$ (entries 7-8). We initially deemed tetrabutylammonium dibutylphosphate $\left(\mathrm{NBu}_{4} \mathrm{PO}_{2}\left(\mathrm{O}^{n} \mathrm{Bu}\right)_{2}\right)$ a suitable base, but it required several days to dry fully once prepared and was inconvenient to handle under ambient atmosphere due to its marked hygroscopicity. To allow a simpler reaction set-up, we sought alternative bases (entries 9-13). Ultimately, we identified potassium $p$-chlorobenzoate as a free-flowing powder that gave satisfactory yields of $\mathbf{2 d}$ despite its low solubility in $\mathrm{PhCF}_{3}$. Although isolated yields were slightly lower in larger vials (entry 14), the increased volume was necessary to conduct the reaction on a scale greater than $0.1 \mathrm{mmol}$. In this work, we opted to report the substrate scope on a $0.2 \mathrm{mmol}$ scale. Control experiments excluding photocatalyst or light failed to generate detectable quantities of $\mathbf{2 d}$, while only trace product was observed in the absence of a base (entries 15-17). 


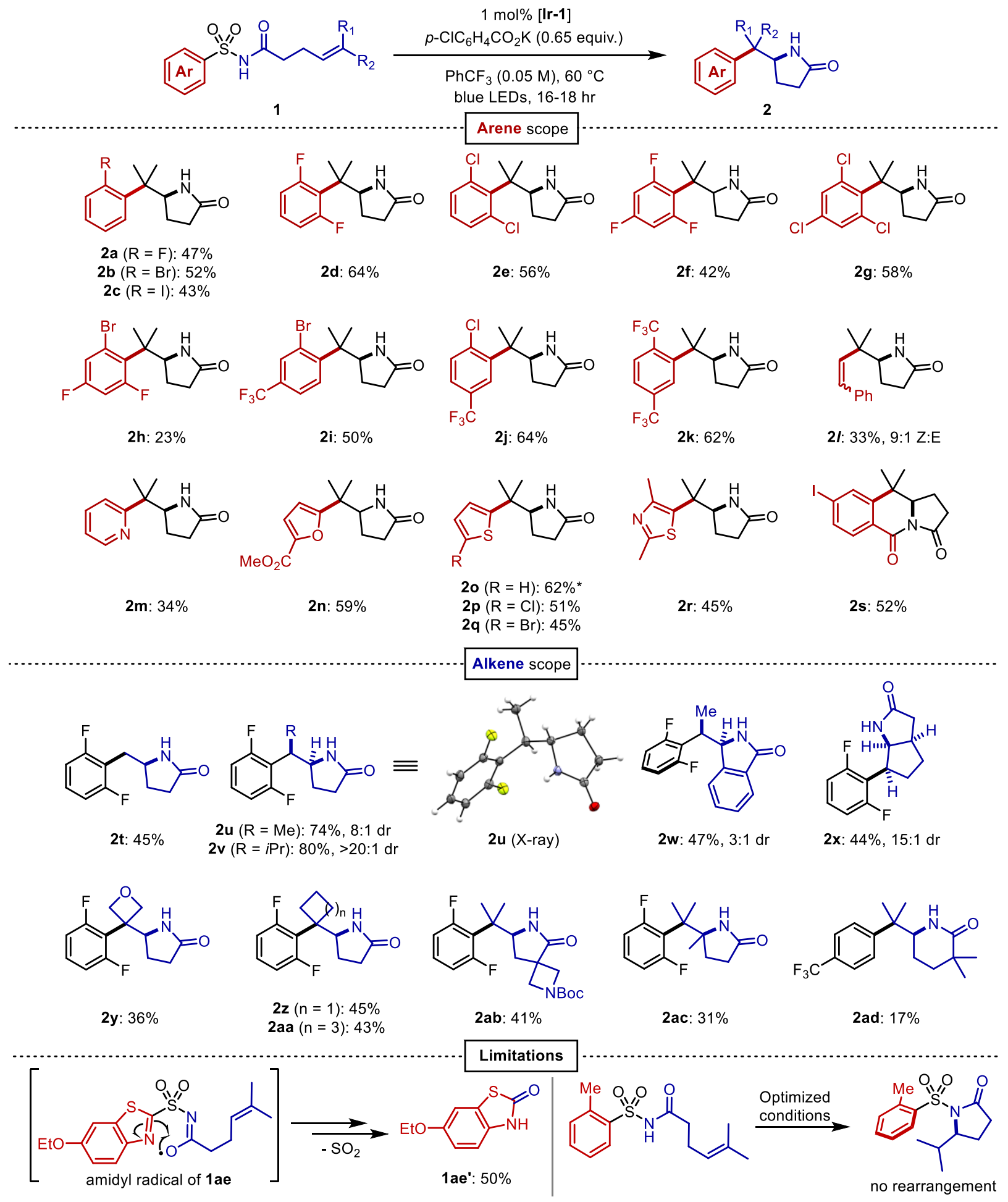

Figure 3. Scope of the aminoarylation. All reactions performed on $0.2 \mathrm{mmol}$ scale. All yields are from isolation. *Isolated as 9:1 mixture with regioisomeric product 20', see Supplementary Information. 


\section{Reaction scope}

After identifying optimal conditions, the arene scope of the reaction was demonstrated on a variety of electron-neutral and electron-deficient mono- and bis-ortho-substituted benzene derivatives, as well as on a selection of heteroaromatic sulfonamides (Figure 3). Orthohalogenated arenes - many of which would be incompatible with palladium or nickel catalystsand ortho-trifluoromethylated arenes were generally well-tolerated (2a-2k). (E)-Styrenyl sulfonamide $1 \boldsymbol{l}$ underwent a vinylogous Smiles-Truce rearrangement and alkene isomerization to give the aminoalkenylation product $\boldsymbol{2} \boldsymbol{l}$ as a 1:9 mixture of $E / Z$ isomers. Heterocycles including pyridine (2m), furan (2n), thiophene (20-2q), and thiazole (2r) could all undergo migration as well in modest to good yields. 2-Substituted thiophene $\mathbf{1 0}$ gave a 9:1 mixture of lactam $\mathbf{2 0}$ with the 3subsituted thiophene regioisomer 20' arising from an unexpected cine-substitution sequence (see Supplementary Information). In the presence of an ortho ester substituent (1s), the amidyl anion liberated upon desulfonylation displaced the alkoxide to produce the tricyclic imide $\mathbf{2 s}$. Next, we surveyed the scope of amenable alkenes. Monosubstituted (2t), disubstituted (2u-2x), trisubstituted (2y-2ab), and tetrasubstituted (2ac) alkenes were all successfully functionalized, producing lactams with diverse carbon skeletons. Seemingly minor manipulations of the alkene tether in 1ad could greatly alter the reaction: when the tether was homologated and gemdimethyl substitution was incorporated alpha to the carbonyl, the ensuing 6-exo trig cyclization triggered the migration of a 4-trifluoromethylphenyl ring lacking ortho substitution. This result was surprising because the same arene in our previous work on dearomative cyclization was not observed to undergo rearrangement. ${ }^{32}$ We believe that the Thorpe-Ingold effect in this substrate accelerates 6-exo-trig ring closure, and that the resultant alkyl radical is oriented closer to the ipso carbon of the sulfonamide than to the ortho carbons. This intriguing divergence invites further study of the impact that conformational biases may exert on the course of the reaction.

Certain limitations also became clear as we interrogated the scope of the reaction. Benzothiazole substrate 1ae degraded to benzothiazolone 1ae' through the aforementioned desulfonylative arene oxygenation, which may be faster than sulfonamidyl radical cyclization in heterocycles with high migratory aptitudes. ${ }^{33}$ Electron-donating ortho substituents on the sulfonamide prohibited the desired aryl transfer. Consequently, hydrogen atom transfer (HAT) or 
reduction of the alkyl radical following 5-exo cyclization led to undesired hydroamination side products.

Compounds $\mathbf{1} \mathbf{u}, \mathbf{1} \mathbf{v}, \mathbf{1} \mathbf{w}$, and $\mathbf{1 x}$ bearing 1,2-disubstituted olefins underwent aminoarylation with varying diastereoselectivities. The major diastereomer of product $\mathbf{2 u}(8: 1 \mathrm{dr})$ was isolated and its relative configuration was elucidated through X-ray crystallographic analysis. A possible model to rationalize the observed stereoselectivity is provided in Figure 4A. Following 5-exo cyclization, a bond rotation positions the larger alkene substituent to minimize steric interaction with the newly formed lactam. When the alkene was substituted with an isopropyl group (1v), compound $\mathbf{2 v}$ was formed as a single diastereomer. In the aminoarylation of N-aroyl sulfonamide $\mathbf{1} \mathbf{w}(1: 1 \mathrm{E} / \mathrm{Z})$, the $\mathrm{sp}^{2}$-hybridized carbon atoms at the lactam/arene ring fusion possess an attenuated steric influence on the methyl group. Therefore, diminished diastereoselectivity (3:1 dr) was observed in the isomer distribution of $\mathbf{2 w}$.

We then investigated the mechanism of $\mathrm{N}$-centered radical generation. We considered three possibilities: (1) oxidation of benzoate by the photocatalyst, followed by HAT from the N-H bond of 1 to the resulting benzoyloxy radical; (2) oxidative MS-CPET involving the photocatalyst and a hydrogen-bonded substrate-benzoate complex, or (3) deprotonation of $\mathbf{1}$ by benzoate and subsequent oxidation of the $N$-acylsulfonamidyl anion. The first proposal seemed unlikely based on observations from the reaction optimization. Specifically, use of potassium o-methylbenzoate as the base gave $55 \%$ yield of product $\mathbf{2 d}$, even though the benzoyloxy radical derived from this compound undergoes 1,5 HAT that would likely outcompete intermolecular HAT in the dilute reaction conditions. ${ }^{34}$ Use of pyridine, with an oxidation potential $\left(2.2 \mathrm{~V}\right.$ vs. SCE in $\mathrm{CH}_{3} \mathrm{CN}$ ) well beyond that of the excited state of Ir-1 (1.68 V vs. SCE in $\mathrm{CH}_{3} \mathrm{CN}$ ), still resulted in $12 \%$ yield of 2d. ${ }^{35,36}$ The second proposal was evaluated and rejected in our previous work based on SternVolmer luminescence quenching experiments, which indicated that tetrabutylammonium dibutylphosphate and $\mathrm{N}$-acylsulfonamides do not form more easily oxidizable complexes in $\mathrm{CH}_{2} \mathrm{Cl}_{2}$ solution. ${ }^{32}$ The base and the solvent examined in the quenching studies differ from those employed in our optimized aminoarylation conditions, but both can be substituted successfully with only moderate yield reduction (see Supplementary Information). To assess the third proposal, we synthesized the putative intermediate $\mathbf{K}^{+}\left[\mathbf{1} \mathbf{d}^{-}\right]$, a deprotonated salt of compound $\mathbf{1 d}$. When a 
A: Diastereoselectivity model

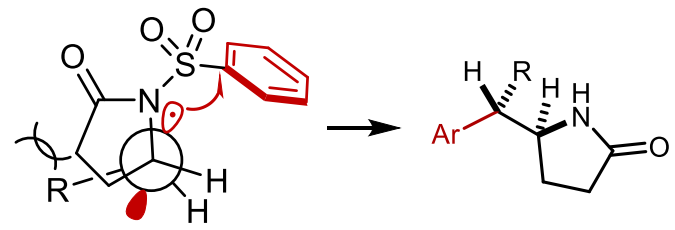

disfavored conformer

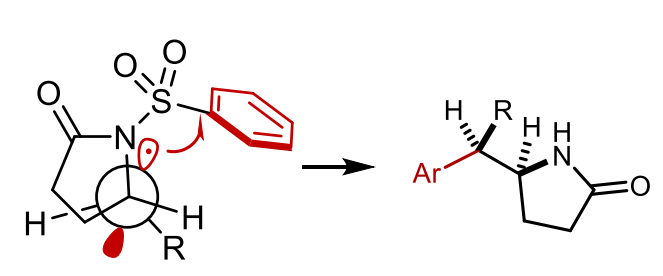

favored conformer major diastereomer

B: Substrate anion reactivity<smiles>CC(C)=CCCC(=O)NS(=O)(=O)c1cc(C(F)(F)F)ccc1Cl</smiles>

$\mathrm{K}^{+}[\mathbf{1 d}]^{-}: 1$ equiv.

$1 \mathrm{j}: 2.3$ equiv.

2d: $73 \%$

2j: $84 \%$
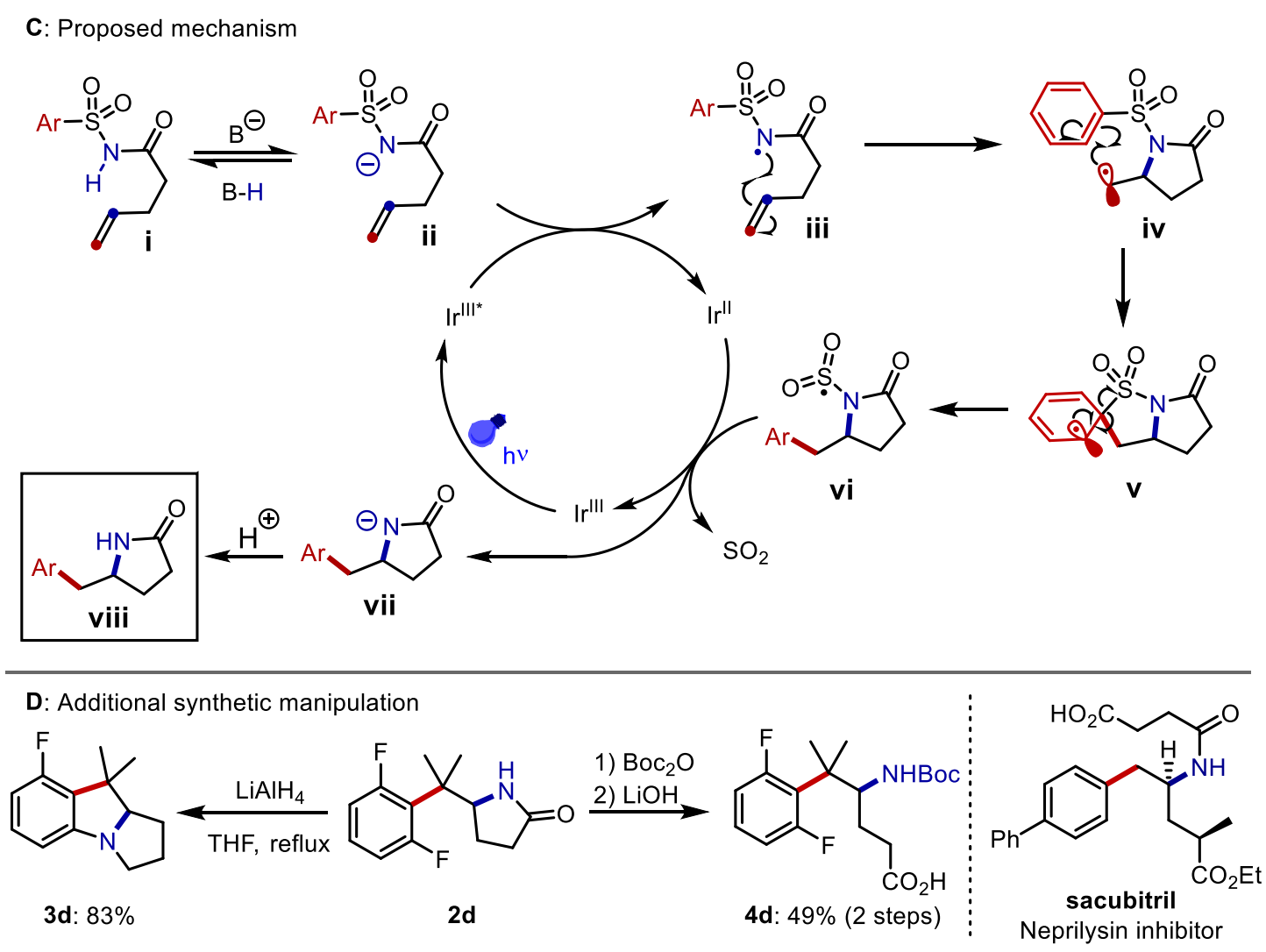

Figure 4. A: Newman projections depicting disfavored and favored conformers of the $\mathrm{N}$-sulfonyl lactam and stereochemical outcomes of $\mathrm{C}-\mathrm{C}$ bond formation B: Experiment establishing reactivity and catalytic turnover of deprotonated $\mathrm{N}$-acylsulfonamides. Yields determined by ${ }^{19} \mathrm{~F}$ NMR integration relative to 4 -fluorobromobenzene internal standard. C: Mechanistic proposal of the aminoarylation. D: Further reactions of aminoarylation products.

3:7 mixture of $\mathbf{K}^{+}\left[\mathbf{1} \mathbf{d}^{-}\right]$and $\mathbf{1} \mathbf{j}$ was subjected to the optimized conditions without added base, we still observed full consumption of both compounds and good yields of their respective products 
2d and $\mathbf{2 j}$ (Figure 4B). These data suggest a stepwise deprotonation-oxidation as the operative mechanism by which the $\mathrm{N}$-centered radical forms. The data also imply that some reaction intermediate can deprotonate the starting material. Based on these findings, we posit a mechanism for the reaction detailed in Figure 4C: The photoexcited iridium catalyst $\mid r^{\|l\|}$ oxidizes the deprotonated $\mathrm{N}$-acylsulfonamide ii to the $\mathrm{N}$-centered radical iii. The $\mathrm{C}-\mathrm{N}$ bond is then formed via 5-exo-trig cyclization and the resultant alkyl radical iv adds to the arene to yield dearomatized spirocycle $\mathbf{v}$. Elimination of the sulfonyl group from $\mathbf{v}$ restores the aromatic system and gives the $N$-sulfonyl radical vi. Desulfonylation from vi and reduction by $1 \mathrm{r}^{\prime \prime}$ restores the ground state of the photocatalyst and produces amidyl anion vii. The anion irreversibly deprotonates either the benzoic acid or another equivalent of $\mathbf{i}$ to furnish the product viii.

Finally, we performed additional diversification of the aminoarylation products that either leveraged the ortho substituents as functional handles to build additional complexity or converted the structures to molecules resembling other biologically active compounds (Figure 4D). Reaction of $\mathbf{2} \mathbf{d}$ with $\mathrm{LiAlH}_{4}$ gave benzopyrrolizidine $\mathbf{3} \mathbf{d}$ through a sequential lactam reduction and $\mathrm{S}_{\mathrm{N}} \mathrm{Ar}$ of fluoride. A Boc protection/hydrolysis sequence yielded 3-(arylmethyl)-3-aminobutyric acid $4 \mathbf{d}$, which mimics the carbon skeleton of the neprilysin inhibitor sacubitril.

\section{Conclusion}

In summary, we have developed a unique alkene aminoarylation that affords products containing privileged arylethylamine connectivity. The method is compatible with a broad selection of unactivated alkenes and is orthogonal to existing cross-coupling methods. We engineered the substrates to curb unproductive pathways en route to an unprecedented SmilesTruce rearrangement prompted by $\mathrm{C}-\mathrm{N}$ bond construction from an $\mathrm{N}$-centered radical. The substrates are easily synthesized from commercially abundant building blocks and the reaction set-up is performed under ambient atmosphere using conveniently handled reagents. The strategy disclosed herein will inform future efforts to revisit the recalcitrant intermolecular variant of this chemistry. This work is a testament to the complexity-building capabilities of $\mathrm{N}$-centered radicals and the cascade reactivities that they can unleash when properly controlled. 


\section{Acknowledgments}

The authors acknowledge financial support for this research from the NIH (GM127774) and the University of Michigan. This material is based upon work supported by the National Science Foundation Graduate Research Fellowship for R.C.M (Grant No. DGE 1256260). We thank Dr. Jeff W. Kampf for collecting and analyzing X-ray crystallography data.

\section{Data availability}

The X-ray crystal structure of compound $\mathbf{2} \mathbf{u}$ is available free of charge from the Cambridge Crystallographic Data Centre under deposition number 2045499.

\section{References}

1. Zhang, A.; Neumeyer, J. L.; Baldessarini, R. J., Recent Progress in Development of Dopamine Receptor Subtype-Selective Agents: Potential Therapeutics for Neurological and Psychiatric Disorders. Chemical Reviews 2007, 107 (1), 274-302.

2. Markowitz, J. S.; Patrick, K. S., The Clinical Pharmacokinetics of Amphetamines Utilized in the Treatment of Attention-Deficit/Hyperactivity Disorder. Journal of Child and Adolescent Psychopharmacology 2017, 27 (8), 678-689.

3. Garvey, W. T., Phentermine and topiramate extended-release: a new treatment for obesity and its role in a complications-centric approach to obesity medical management. Expert Opinion on Drug Safety 2013, 12 (5), 741-756.

4. $\quad$ Lippa, R. A.; Battersby, D. J.; Murphy, J. A.; Barrett, T. N., Synthesis of Arylethylamines via C(sp3)C(sp3) Palladium-Catalyzed Cross-Coupling. The Journal of Organic Chemistry 2021, 86 (4), 3583-3604.

5. Nguyen, T. M.; Manohar, N.; Nicewicz, D. A., anti-Markovnikov Hydroamination of Alkenes Catalyzed by a Two-Component Organic Photoredox System: Direct Access to Phenethylamine Derivatives. Angewandte Chemie International Edition 2014, 53 (24), 6198-6201.

6. Ryu, J.-S.; Li, G. Y.; Marks, T. J., Organolathanide-Catalyzed Regioselective Intermolecular Hydroamination of Alkenes, Alkynes, Vinylarenes, Di- and Trivinylarenes, and Methylenecyclopropanes. Scope and Mechanistic Comparison to Intramolecular Cyclohydroaminations. Journal of the American Chemical Society 2003, 125 (41), 12584-12605.

7. Utsunomiya, M.; Kuwano, R.; Kawatsura, M.; Hartwig, J. F., Rhodium-Catalyzed Anti-Markovnikov Hydroamination of Vinylarenes. Journal of the American Chemical Society 2003, 125 (19), 5608-5609.

8. Utsunomiya, M.; Hartwig, J. F., Ruthenium-Catalyzed Anti-Markovnikov Hydroamination of Vinylarenes. Journal of the American Chemical Society 2004, 126 (9), 2702-2703.

9. Boyington, A. J.; Seath, C. P.; Zearfoss, A. M.; Xu, Z.; Jui, N. T., Catalytic Strategy for Regioselective Arylethylamine Synthesis. Journal of the American Chemical Society 2019, 141 (9), 4147-4153.

10. Choi, G. J.; Knowles, R. R., Catalytic Alkene Carboaminations Enabled by Oxidative Proton-Coupled Electron Transfer. Journal of the American Chemical Society 2015, 137 (29), 9226-9229.

11. Miller, D. C.; Choi, G. J.; Orbe, H. S.; Knowles, R. R., Catalytic Olefin Hydroamidation Enabled by Proton-Coupled Electron Transfer. Journal of the American Chemical Society 2015, 137 (42), 13492-13495.

12. Morris, W. D.; Mayer, J. M., Separating Proton and Electron Transfer Effects in Three-Component Concerted Proton-Coupled Electron Transfer Reactions. Journal of the American Chemical Society 2017, 139 (30), 10312-10319.

13. Chu, J. C. K.; Rovis, T., Amide-directed photoredox-catalysed C-C bond formation at unactivated sp3 C-H bonds. Nature 2016, 539 (7628), 272-275. 
14. Ney, J. E.; Wolfe, J. P., Palladium-Catalyzed Synthesis of N-Aryl Pyrrolidines from $\mathrm{Y}$-(N-Arylamino) Alkenes: Evidence for Chemoselective Alkene Insertion into Pd - N Bonds. Angewandte Chemie International Edition 2004, 43 (27), 3605-3608.

15. Ney, J. E.; Wolfe, J. P., Selective Synthesis of 5- or 6-Aryl Octahydrocyclopenta[b]pyrroles from a Common Precursor through Control of Competing Pathways in a Pd-Catalyzed Reaction. Journal of the American Chemical Society 2005, 127 (24), 8644-8651.

16. Liu, Z.; Wang, Y.; Wang, Z.; Zeng, T.; Liu, P.; Engle, K. M., Catalytic Intermolecular Carboamination of Unactivated Alkenes via Directed Aminopalladation. Journal of the American Chemical Society 2017, 139 (32), 11261-11270.

17. Taeho, K.; Nana, K.; Peter, C.; Hao, Z.; Klement, F.; Keary, E., Nickel-Catalyzed 1,2-Carboamination of Alkenyl Alcohols. ChemRxiv 2021.

18. Zheng, S.; Gutierrez-Bonet, A.; Molander, G. A., Merging Photoredox PCET with Nickel-Catalyzed Cross-Coupling: Cascade Amidoarylation of Unactivated Olefins. Chem 2019, 5 (2), 339-352.

19. Tada, M.; Shijima, H.; Nakamura, M., Smiles-type free radical rearrangement of aromatic sulfonates and sulfonamides: syntheses of arylethanols and arylethylamines. Organic \& Biomolecular Chemistry 2003, 1 (14), 2499-2505.

20. Whalley, D. M.; Duong, H. A.; Greaney, M. F., A visible light-mediated, decarboxylative, desulfonylative Smiles rearrangement for general arylethylamine syntheses ${ }^{+}$. Chem. Commun 2020, 56, 11493-11493.

21. Monos, T. M.; McAtee, R. C.; Stephenson, C. R. J., Arylsulfonylacetamides as bifunctional reagents for alkene aminoarylation. Science 2018, 361 (6409), 1369-1373.

22. Nicewicz, D.; Roth, H.; Romero, N., Experimental and Calculated Electrochemical Potentials of Common Organic Molecules for Applications to Single-Electron Redox Chemistry. Synlett 2015, 27 (05), 714-723.

23. Wang, L.; Wu, F.; Chen, J.; Nicewicz, D. A.; Huang, Y., Visible-Light-Mediated [4+2] Cycloaddition of Styrenes: Synthesis of Tetralin Derivatives. Angewandte Chemie International Edition 2017, 56 (24), 68966900.

24. Zhu, Q.; Graff, D. E.; Knowles, R. R., Intermolecular Anti-Markovnikov Hydroamination of Unactivated Alkenes with Sulfonamides Enabled by Proton-Coupled Electron Transfer. J Am Chem Soc 2018, 140 (2), 741-747.

25. Ji, Y.-X.; Li, J.; Li, C.-M.; Qu, S.; Zhang, B., Manganese-Catalyzed N-F Bond Activation for Hydroamination and Carboamination of Alkenes. Organic Letters 2020, 23.

26. Zeng, W.; Chemler, S. R., Copper(II)-Catalyzed Enantioselective Intramolecular Carboamination of Alkenes. Journal of the American Chemical Society 2007, 129 (43), 12948-12949.

27. Kaneko, K.; Yoshino, T.; Matsunaga, S.; Kanai, M., Sultam Synthesis via Cu-Catalyzed Intermolecular Carboamination of Alkenes with N-Fluorobenzenesulfonimide. Organic Letters 2013, 15 (10), 2502-2505.

28. Wang, D.; Wu, L.; Wang, F.; Wan, X.; Chen, P.; Lin, Z.; Liu, G., Asymmetric Copper-Catalyzed Intermolecular Aminoarylation of Styrenes: Efficient Access to Optical 2,2-Diarylethylamines. Journal of the American Chemical Society 2017, 139 (20), 6811-6814.

29. Wang, X.; Xia, D.; Qin, W.; Zhou, R.; Zhou, X.; Zhou, Q.; Liu, W.; Dai, X.; Wang, H.; Wang, S.; Tan, L.; Zhang, D.; Song, H.; Liu, X.-Y.; Qin, Y., A Radical Cascade Enabling Collective Syntheses of Natural Products. Chem 2017, 2 (6), 803-816.

30. Chen, J.; Guo, H. M.; Zhao, Q. Q.; Chen, J. R.; Xiao, W. J., Visible light-driven photocatalytic generation of sulfonamidyl radicals for alkene hydroamination of unsaturated sulfonamides. Chem Commun (Camb) 2018, 54 (50), 6780-6783.

31. Lassalas, P.; Gay, B.; Lasfargeas, C.; James, M. J.; Tran, V.; Vijayendran, K. G.; Brunden, K. R.; Kozlowski, M. C.; Thomas, C. J.; Smith, A. B.; Huryn, D. M.; Ballatore, C., Structure Property Relationships of Carboxylic Acid Isosteres. Journal of Medicinal Chemistry 2016, 59 (7), 3183-3203. 
32. McAtee, R. C.; Noten, E. A.; Stephenson, C. R. J., Arene dearomatization through a catalytic Ncentered radical cascade reaction. Nature Communications 2020, 11 (1), 2528.

33. Zhang, H.; Kou, L.; Chen, D.; Ji, M.; Bao, X.; Wu, X.; Zhu, C., Radical-Mediated Distal Ipso-Migration of O/S-Containing Heteroaryls and DFT Studies for Migratory Aptitude. Organic Letters 2020, 22 (15), 59475952.

34. Wang, J.; Tsuchiya, M.; Tokumaru, K.; Sakuragi, H., Intramolecular Hydrogen-Atom Transfer in 2Alkylbenzoyloxyl Radicals as Studied by Transient Absorption Kinetics and Product Analyses on the Photodecomposition of Bis(2-alkylbenzoyl) Peroxides. Bulletin of the Chemical Society of Japan 1995, 68 (4), 1213-1219.

35. Appendix B: Tables of Physical Data. In Fundamentals and Applications of Organic Electrochemistry, Fuchigami, T.; Inagi, S.; Atobe, M., Eds. 2014; pp 217-222.

36. Choi, G. J.; Zhu, Q.; Miller, D. C.; Gu, C. J.; Knowles, R. R., Catalytic alkylation of remote C-H bonds enabled by proton-coupled electron transfer. Nature 2016, 539 (7628), 268-271. 